Safety Clutter: The accumulation and persistence of 'safety' work that does not contribute to operational safety

\author{
A.J. Rae ${ }^{a *}$, D. J. Provan ${ }^{a}$, D. E. Weber ${ }^{\mathrm{a}}$ and S. W. A. Dekker ${ }^{\mathrm{a}}$ \\ ${ }^{a}$ Safety Science Innovation Lab, Griffith University, Brisbane, Australia \\ *corresponding author \\ d.rae@griffith.edu.au \\ +61450161361
}

170 Kessels Rd, Nathan, Qld, 4111

Funding Details: This work was supported by Downer Group, and the Logan Water Infrastructure Alliance. 


\section{Safety Clutter: The accumulation and persistence of 'safety' work that does not contribute to operational safety}

In this paper we provide a description of a previously unlabelled and under-theorised problem in safety management - 'safety clutter'. Safety clutter is the accumulation of safety procedures, documents, roles and activities that are performed in the name of safety, but do not contribute to the safety of operations. Safety clutter is a problem because of the opportunity cost of ineffective activity, because clutter results in cynicism and 'surface compliance,' and because clutter can hamper innovation and get in the way of getting work done. We identify three main mechanisms that generate clutter: duplication, generalisation, and over-specification of safety activities. These mechanisms in turn are driven by asymmetry between the ease and opportunity of adding or expanding safety activities, and the difficulty and lack of opportunity for reducing or removing safety activities. At the end of the paper we provide some concrete suggestions for reducing safety clutter, based on our analysis of the problem.

Keywords: safety clutter; bureaucracy; safety management systems

Subject classification codes:

\section{Introducing and defining safety clutter}

\section{The problem of clutter}

When 'safety' rules impose a significant and unnecessary burden on the performance of everyday activities, both work and safety suffer. Here is a real-world story from one of our researchers to illustrate the nature and extent of the problem.

While I was asking supervisors about which safety practices they liked or disliked, they frequently mentioned problems with 'induction'. On further inquiry it appeared that contractors were required to complete an online induction process that was intended to take three hours, but could take up to six hours for a contractor with low computer literacy. This was only part of the picture, though. As I explored further, it became clear that the term 'induction' sometimes referred to the online induction, and sometimes to site inductions. Eventually, I realised that there were five separate inductions, each covering roughly the same relevant material, and a variable amount of irrelevant material. Before performing work on a site, a 
contractor could be required to complete all five inductions - amounting to more than a full day of work. At least on paper, these inductions were required even for a worker spending only a few hours on site.

In an effort to reduce the cost of providing inductions, another organisation introduced Computer-Based Training (CBT) inductions. Given the work to be done, not all contractors were entirely literate. One of the contractors became known as the 'super-inductor'. Their employee would sit at seven computer terminals at the same time, rolling back and forth between them, and making sure every contractor passed the induction. Few of them ever found out what was actually in the induction, but they'd passed and were (at least on the record) 'safe' to be on site.

Not every project requires five inductions, but every organisation has safety activities that are at times performed with no expectation that they provide any real safety benefit. Such activities drain time, resources and attention that could be spent on improving the safety of operational work. They also create and perpetuate harmful beliefs about safety. In such cases we suggest that safety outcomes can be improved by reducing the amount of safety activity. An unquestionable example of this is system duplication, where the same people conduct the same activity twice, using differently formatted paperwork. This is not limited to so-called hard-hat industries. Surgeons, too, have complained of 'checklist fatigue' and a lack of buy-in. They have remarked that if any more checklists would be instituted, they 'would need a checklist for all the checklists’ (Stock \& Sundt, 2015).

All of this comes with a greater trend toward bureaucratization and risk aversion, as well as centralization, standardization and increased surveillance in safety (Dekker, 2014b; Wears \& Hunte, 2014).

\section{The Definition of clutter}


The purpose of this paper is to provide a theoretical and practical description of 'safety clutter'. We define safety clutter as 'the accumulation of safety procedures, documents, roles and activities that are performed in the name of safety, but do not contribute to the safety of operational work.'

This basic definition conceals several underlying epistemological and ontological questions. By what standard can we judge whether something 'contributes to safety'? Who gets to say whether something is or is not clutter? For each safety activity, we might imagine three dimensions:

(1) Contribution: the extent to which the activity has safety value

(2) Confidence: the certainty (either through evidence or strength of belief) with which this judgement is made

(3) Consensus: the level of agreement about the safety value of the activity between those who mandate the activity, those who perform the activity, and those who are ostensibly kept safe by the activity

There is a contestable boundary between what is and is not clutter, based on how the evidence of efficacy for any individual item is evaluated. There is also a grey area between clutter and not-clutter where items have a safety benefit under some circumstances, but are frequently applied in circumstances or ways where this benefit is not realised. Checklists are an example of a practice in this grey area. There is considerable evidence that they can be very effective, but also evidence that they are sometimes not effective at all (Leape, 2014).

\section{Questions about Clutter}

In this paper we seek to explain the core phenomenon of clutter, rather than to explore edge cases. We are concerned with safety activities that do not contribute at all to 
operational safety, and where there is strong evidence or a strong belief held by those who perform the activities that this is the case.

The principal research question addressed by this paper is:

(1) What is the nature of safety clutter?

This question is divided into the sub-questions:

(2) What are the mechanisms that create clutter? (Section 3)

(3) What causes safety clutter and makes it hard to remove? (Section 4)

(4) What are the effects of safety clutter? (Section 5)

(5) What can we do about safety clutter? (Section 6)

Safety clutter is a newly theorised phenomenon, and so this paper does not contain a formal literature review. In Section 7 we provide a link between our explanation of safety clutter and existing theories of safety bureaucracy and institutional work.

\section{Investigating safety clutter}

\section{Source data for finding clutter}

The description of safety clutter in this paper is drawn mainly from four separate projects. The primary findings of each project have been or are being reported elsewhere, but each project also shed light on the safety clutter problem.

The first project (Study 1), the Woolworths Project (Dekker, 2017), was a large scale, condition-controlled experiment comparing safety in thirty supermarkets in Queensland, Australia, over a period of twelve months. The supermarkets were divided into three experimental conditions: a neutral control condition under current rules, a 
condition in which all head office safety rules were removed, and condition in which rules were removed and store leadership was facilitated to plan their own safety. The project included in-store observations and conversations by two researchers over the duration of the experiment, and many conversations with store managers and the organisation safety team about the effectiveness of safety practices.

The second project (Study 2) was a longitudinal study of the identity and practice of safety practitioners within an Australian energy extraction, production and distribution company (Provan, Dekker, \& Rae, 2018). Twelve practitioners took part in an in-depth initial interview and then monthly reflective interviews about their goals, activities, and motivations.

The third project (Study 3) was a qualitative analysis of 'Authority to Stop' policies and practices in the same organisation as the second project (Weber, Macgregor, Provan, \& Rae, 2018). This study consisted of focus group sessions with 34 experienced workers and managers at several gas terminals. Participants provided insight into the reasons to stop work, the factors that support and hinder the stopping of work, and ways in which stopping takes place.

The fourth project (Study 4) was in a water infrastructure construction and renewal business. This paper is the first output from Study 4 . The purpose of the study was to identify ineffective and unpopular safety practices, in support of a follow-on project to test the effect of removing those practices. The study involved one-hour interviews with a cross-section of the business, ranging from the manager of the business through to supervisors employed by subcontractors. 
In each of these projects, we took the part of engaged participants, working with the organisation to make sense of current practices, and to plan future improvements. As participants we observed first-hand meetings about changing, reducing and testing safety practices. We watched managers and safety practitioners grappling with the social, psychological and political difficulty of removing or changing safety rules - even when they themselves had already argued that the rules were ineffective and counterproductive.

In addition to the four main studies, we have included observations from our own industrial experiences, or from other projects. Examples from the main studies are marked in the text as (Study X). Other examples that we have directly observed are marked as (Researcher observation).

\section{Method for analysing clutter}

This paper was created following the precepts of grounded-theory analysis. The researchers started with a topic of interest - safety clutter - and developed theory simultaneously with data collection through a process of constant comparison between new data and emergent theory. Within grounded theory there are two main approaches, 'Glaserian' or 'Straussian' (Howard-Payne, 2015). The critical-realism ontology of Glaser would be inappropriate in this paper unless we could "embody the role of objectively detached observers" (Howard-Payne, 2015). We could not (Dekker, 2017, 2014b; Provan, Dekker, \& Rae, 2017; Rae \& Alexander, 2017). As these references show, the authors were, in the language of McGhee et al (2007) "theoretically sensitized and familiar with the literature on the topic" at the start of the study.

We therefore adopted a Straussian approach in which we recognised and acknowledged our own pre-existing conceptions of clutter - in particular about what did 
and did not 'count' as clutter - and explicitly tested that initial understanding against our observations during the study, and the statements made by participants.

Two different units of analysis featured in this study. The first unit were statements about, or observations of, specific safety activities and artefacts. These provided specific examples of clutter, from which generalised characterisations could be formed. The second unit were statements about the phenomenon of clutter. These statements were often elicited by presenting tentative conclusions about clutter to research participants, and seeking their response.

\section{Characterising Safety Clutter}

In this section, we describe safety clutter according to the mechanism by which an activity becomes clutter and can be recognised as clutter. These mechanisms are proximate causes of clutter, but they are insufficient to explain why clutter exists and persists. In section 4 we provide a broader theory for the cause of clutter.

\section{Clutter by Duplication}

'Duplication' is where two or more very similar activities fill the same safety function, and where the duplicate activities add no additional safety. Clutter by duplication is not the same as intentional and desirable reproduction. For example, an organisation might deliberately perform a safety-critical check by two different people as a form of redundancy, or might repeat an activity at set intervals as a form of monitoring. Redundancy and monitoring, used appropriately, can increase safety.

\section{Inter-organisational Duplication}

Inter-organisational duplication occurs when more than one organisation governs the exact same operational activity. This may occur when: 
- An activity is performed by a contracted organisation, but where a principal or client (or both) require the use of their own safety systems (Study 2, 3, 4 and Researcher observation)

- An activity falls under the jurisdiction of two different regulators, with similar but not identical requirements (Researcher observation)

In such cases either the governing legislation or the organisations themselves may have rules to establish which system should apply. This fails to prevent duplication if there is conflicting or ambiguous guidance on which system to follow, or if both parties insist on their own system being followed.

An example of inter-organisational duplication is the issuing of permits for hazardous work. A permit is typically a document that describes how the work is to be conducted, signed by a manager from the permitting organisation. The purpose of a permit is to ensure that hazardous work does not proceed without appropriate planning and safety controls. We found several instances where two almost-but-not-quite identical documents would be prepared, for checking by managers from two different companies (Study 4, Researcher observation).

\section{Multiple-relationship Duplication}

Multiple-relationship duplication occurs when one organisation (typically a contractor) does business with multiple clients. This may occur when:

- An organisation works with many different companies, each with similar but differently administered processes (Study 4).

- An organisation works with multiple business units from within a single parent organisation, each with different processes (Study 2). 
In study 2, different parts of the same organisation had separate procurement processes with different contractor safety pre-qualification systems. One contractor who worked for both parts of the organisation had to: complete two pre-qualifications, submit two safety plans, be subject to two auditing programs, and submit two safety reports.

\section{Inter-system Duplication}

Inter-system duplication occurs when multiple management systems are used within the same organisation, often as part of a nominally integrated business management system. Duplication occurs when policies or technical standards addressing separate concerns apply to a single activity in a similar way. Safety systems frequently overlap with:

- Procurement systems (Study 1, 2, 3, 4 and personal observation);

- Human resource systems (Study 1);

- Environment management systems (Study 2, 3 and 4); and

- Project management systems (Study 2, 3, and 4)

An example of inter-system duplication is training. The organisation in Study 3 operated two separate training systems; one system was for safety training, and the other was administered by HR for all other training. Induction training programs included content from both systems. Two separate attendance forms had to be completed for the same training session, and sent to two different administrative departments.

\section{Within-system Duplication}

Duplication within a single system occurs in two ways. Firstly, the system may be 
designed with excessive layers of checks and approvals. Multiple checks at first may appear to be a form of intentional redundancy, or of functional allocation where each check serves a different purpose. However, the original purpose of the checks can be forgotten, or degraded because each checker relies on the other checks. Secondly, the system may contain obsolescent process.

For example, the organisation in Study 2 had a 'journey management system' to monitor where employees planned to drive, and when they planned to arrive at locations. This required filling out forms, having the forms signed by a supervisor, and then phoning a call centre to record the information in the form. This already involved an unnecessary layer (filling out the form, and then the call centre filling out a matching record). The organisation fitted an electronic In-Vehicle Monitoring System (IVMS) to all of their vehicles, which provided an automatic centralised record of where each vehicle was, and who was driving. The original process was not removed with the introduction of the IVMS.

A specific mechanism for within-system duplication is mismatch between the importance of a safety matter and the level of the company hierarchy that deals with the matter. Whilst some theories of safety culture argue for 'strong' leadership involvement in safety, in practice if one level of a hierarchy is responsible for an issue, then every layer below that issue must also be involved, providing reports or delegating instructions.

In both Study 2 and Study 3 we observed this occurring in response to safety incidents. In Study 3 a safety issue associated with a customer site was reported up three levels of management. The person reporting the issue had to explain the incident not only to the call centre operator (who was actually responsible for resolving the issue 
with the customer) but also received phone calls from each level of management asking for the same description of the issue.

\section{Industry Standardisation}

Industry standardisation is where an industry (e.g. oil and gas, construction) gets together to jointly solve safety problems. Many high safety risk industries have national and international safety forums consisting of senior managers of the individual member companies. When the industry agrees that is has a common safety problem, and develops a shared solution then each of the companies agrees to implement this within their organisation for the benefit of the industry. This leads to a duplication of existing processes.

For example, the industry in Study 2 wanted to evaluate its safety culture with the intention of making industry-wide improvements. Individual organisations required all of their employees to complete the industry safety culture survey, even though they completed their company's own safety culture questionnaire one week before.

The industry in Study 4 experienced a similar problem with inductions. They developed a common competency standard so that subcontractor employees could obtain a 'card' rather than attending training for each new principal contractor. Each principal contractor continued to hold their own training as before, along with a process for checking and recording the cards.

\section{Clutter by Generalisation}

'Generalisation' is where requirements that make sense in one situation are applied across many or all situations. This results in an operational activity being governed by rules or processes that are irrelevant for the performance of the activity. 


\section{Conservatism}

Conservatism is where a high-level rule allows for discretion, but the rule is applied at lower levels with the discretion removed. Conservatism is the opposite of risk-based decision making, operating on the assumption that the strictest requirements always provide the most safety.

Examples of conservatism include:

- Company rules that suggest a specific form of protection should be used 'when appropriate' are implemented by a subcontractor to require the protection at all times (e.g. in Study 3, office workers in administration buildings outside of hazardous areas were required to wear full protective clothing and safety boots);

- Regulatory requirements intended especially for 'high risk' work applied to all work in a business unit that performs some high-risk work (e.g. in Study 2 and Study 4, workers completed a work method statement for all jobs, not just for high-risk construction activities);

- Company rules that provide a set of options interpreted by a local business unit to require every item in the set (e.g. in Study 3, sales and office based teams were required to complete training and assessments in high-risk work e.g. confined space entry, and working at heights).

Clutter by over-generalisation creates the problem of indifferentiation. It is no longer clear to individuals or the organisations which are the dangerous activities or locations where organisational resources need to be focussed. For example, in Study 2 all work tasks at a hazardous site required a work permit, expanded from the previous requirement that tasks impacting on safety critical systems or with the risk of fatality required a permit. The system then no longer differentiated between the process 
required to perform hot-work in potentially explosive atmospheres, and the process for cleaning the lunch room. So much additional work was introduced with the same resource levels that the quality of the outputs for all permits suffered.

\section{Symbolic Application}

Symbolic application is where a rule intended for high-risk operational activities is applied in administrative spaces and organisational units as a gesture or ritual to indicate that the entire company takes safety seriously. Symbolic application is a special case of clutter, because whilst everyone who takes part knows that the specific instances of the activity have no value, there may be meta-belief that universal enactment of symbolic safety will ultimately contribute to the safety of operational activities.

Symbolic application can operate directly, as in the case of 'toolbox talks' for office workers, or indirectly, through symbolic or humorous uses of reporting systems that are treated equivalently to serious operational incidents, as in the case of dirty dishes in the office kitchen (Study 2). Such symbolic application may be responsible for otherwise inexplicable instances of 'OHS gone mad':

- Signs on stairs instructing workers to 'maintain 3 points of contact' taken from safety rules for the use of ladders (Study 1);

- Duplicate labels warning users that a hot tap will issue hot water taken from hazardous area warning labels (Studies 1 and 4); and

- Risk assessments for travel in commercial passenger vehicles taken from managing hazardous field activities (Researcher observation).

\section{Attempted simplification}

Attempted simplification is where a blanket rule is created to avoid the need for caseby-case decision making. The rule may be justified because it is: 
- cognitively simpler - workers can apply the rule without evaluating the context;

- $\quad$ socially simpler - the same rule applies to everyone and all circumstances; or

administratively simpler - managers can identify violations without needing to check if there is a local rationale for not following the rule. Examples of attempted simplification include a rule in Study 4 to treat every 'manhole' as a confined space, requiring preparation of a rescue winch before access, and a rule in Study 1 that required each store to hold the exact same number of non-slip entrance mats, regardless of the number of entrances.

Another example is the compilation and promulgation of 'safety essentials', 'life-saving rules', or 'golden rules' (Studies 2, 3, 4 and Researcher Observation). Some workers may never experience the situations in which some of the 'essential' rules apply, but all workers are required to know and follow all of the rules. A consequence of this may be managers required to inspect, enforce and report that an inapplicable rule is 'being followed' - for example that office cleaners are trained and certified to operate all equipment and machinery.

\section{Least-common-denominator}

'Least common denominator' is where a safety practice is intended to support workers who are inexperienced, or have an unsophisticated understanding of safety. Such practices offer no benefit to more capable employees, and may appear belittling, patronising and disenfranchising.

An example of a 'least common denominator' practice is mandatory safety training, designed to suit workers with no knowledge of safety principles or practices, rolled-out to every employee, including experienced and post-graduate educated safety professionals (Study 4). A more specific example is a rule that any use of a chainsaw requires a permit and personal observation from a supervisor (Study 4). Such a rule is 
relevant for preventing casual and inappropriate use of a potentially dangerous tool, but offers no benefit to an experienced arborist who uses chainsaws on a daily basis.

'Least common denominator' can apply to organisations as well as individuals. Processes designed to manage unfamiliar or inexperienced subcontractors are often unsuitable for 'mature' subcontractors with their own safety management systems, or where there is a strong partnership existing between the principal contractor and the subcontractor (Study 4 and Researcher observation).

\section{Clutter by Over-specification}

'Over-specification' is the unnecessary translation of good practice into documented process. There are three problems associated with over-specification. Firstly, the creation of supporting documents increases the work associated with the practice, by adding secondary tasks (reading documents and filling out forms). In situations with limited time or attention, the additional work may detract or distract from the primary task. Secondly, documented processes are more vulnerable to 'requirements creep', where a simple practice becomes more complicated through the addition of contingencies and guidance for every possible concern or situation. Thirdly, processes are commonly over-prescriptive. A task may be able to be completed in a number of ways to match different work contexts, but one particular method becomes the only acceptable means of compliance.

An example of over-specification is the creation of cards, forms, and procedures to formalise workplace risk assessments (Studies 1, 2, 3 and 4). It is good practice for workers to check for nearby hazards before starting a potentially dangerous task. The creation of a checklist to formalise this process makes the risk assessment more complicated by requiring a form to be filled out, collected, and checked. The form itself may become more complicated over time as safety personnel or managers come up with 
new hazards that should be considered. It may be mandatory for the risk assessment to be completed at the job-site, immediately before the task, which then provides restrictions on the ability to involve additional stakeholders or to complete the process earlier in the planning stage of the work to improve its effectiveness.

\section{Explaining safety clutter}

In the previous section we provided a description of the forms and immediate causes of safety clutter. Here, we will present a theory for why safety clutter is prevalent.

At the heart of our theory are two asymmetries:

(1) It is easier to add or expand safety work, than to remove or reduce safety work.

(2) There are many regular or ad-hoc events that trigger the addition or expansion of safety work, but relatively few opportunities to remove or reduce safety work.

Together, these asymmetries create a 'ratchet effect' whereby the number and complexity of safety activities in an organisation increases over time. Even deliberate attempts to 'simplify' safety are likely to result in excessive generalisation (i.e. more clutter) rather than a genuine reduction in safety activity (Study 1 and Study 4).

The two asymmetries, and the resulting ratchet effect, were observed in all four studies, and confirmed by multiple participants in each organisation. The underlying causes are less directly evidenced, and represent the researchers' interpretation of our observations. Often the evidence for these causes lies in the discourse - how participants spoke about activities and events, rather than the factual content of the statements. To strengthen our argument, we have linked to other published evidence and discussion where applicable. We suggest that the asymmetries are driven by a range of epistemological, psychological, organisational, regulatory and professional causes. 
The causes include, as a non-exhaustive list: :

(1) Responses to accidents and incidents that trigger additional safety work

(2) A need to demonstrate to others that we are managing safety

(3) The separation and professionalisation of the safety role

(4) Compliance with goal-based regulatory regimes

Each of the above four factors can, under the right circumstances, contribute significantly to the safety of operational work. However, organisations and the individuals within them feel constant pressure to improve the safety of their workplaces. In their attempt to improve safety, clutter is often introduced. Once it is present in the organisation, it persists, leading to a gradual accumulation of clutter.

\section{Responses to accidents and incidents that trigger additional safety work}

Clutter is caused by the social and psychological need to take action in response to unplanned and unwanted events and circumstances, particularly those that relate to the safety of workers. Organisations need to respond quickly to these disruptions to maintain continuity in their activities. Individuals need to feel that they have regained control over things that the feel responsible or accountable for. In this environment, where there has been clear and present danger to people, new safety activities get introduced quickly. The response may fall short of fixing the actual problem, and instead introduce additional safety work into the organisation with no benefit to the safety of operational work. Safety clutter is caused by this focus on quickly resolving moments of crises and psychological uncertainty, rather than the long-term effectiveness of solutions. 
It is not even always necessary to have an accident or incident - 'What if?' thinking can be sufficient to trigger an uncritical safety response. Additional safety work is defended based on 'What if there was an accident?', rather than 'What is the evidence for this particular way of doing things?'. The potential severity of the consequences creates a greater fear of not doing something that might be perceived in hindsight as 'should have been done', than fear of inefficiency or waste of time. This is helped by the fact that it's often not the individuals who are determining the safety work who are the ones that are required to implement or comply with it. Individual safety activities or requirements have an apparently low cost compared to the 'worst case scenario' of an accident that could threaten the survival of the company.

Investigations almost always add or expand safety work, and rarely if ever replace or remove existing activities. The organisation that has had the accident is viewed as 'not having done enough' for safety, so the rational response is to add additional safety activities. The need to 'do more safety' can become the dominant driver rather than the need to 'improve safety'.

Where does the new safety work come from? Additional safety work gets created and copied. The creation of additional work results from managers and safety practitioners expanding existing processes or creating new administrative safety activities. More commonly, organisations compare themselves with other companies that didn't have the accident to 'learn' what they are doing differently or additionally for safety, and then copy.

\section{The need to demonstrate that safety is managed}

Organisations have a large and diverse group of stakeholders that are interested 
in, and can impact their sustainability and success. Stakeholders include: governments, shareholders, customers, business partners, communities, and employees. Increasingly, organisations need to demonstrate to each of these stakeholders that they care about and are managing the safety risks associated with their activities.

Where work is already safe, the additional need to 'demonstrate safety' is, almost by definition, not contributing to the safety of work. Worse, the need to demonstrate safety can drive a feedback loop where the very act of adding new activities is proof that the organisation cares about safety. Adding safety work and activity shows care; removing safety work, even if it is safety clutter, can be interpreted as a lack of care.

In many industries and companies, major safety accidents and incidents are rare, and so the absence of safety incidents themselves is not sufficient to demonstrate safety. Safety scholars reinforce the message that 'the absence of safety incidents is not evidence of safety' (Manuele, 2009). One solution is to use the volume of safety activity as a proxy for the physical level of safety in the organisation. Administrative activities - including activities that document and count other activities - create a paper trail of safety evidence that can be used to 'demonstrate' safety to stakeholders. Ironically, very effective safety activities may be unhelpful for demonstrating safety (because they leave no paper trail) whereas safety clutter provides tangible 'evidence' of safety.

Safety demonstration is not just about appeasing external stakeholders. It is a basic human motivation to perform, and be seen to perform, valuable work (Pink, 2011). A manager might have a subtle and positive influence over safety, and yet have 
'nothing to show' for the time they have spent. Administrative safety activity provides a record of time spent, people spoken to, forms filled out and processes completed.

Bourrier and Bieder (2013) observed that the only way to be seen to create greater safety - if not by changing or adding a new piece of technology — is to write more rules, to create more procedures, or to demand more compliance.

\section{The separation and professionalisation of the safety role}

Since the early 1980s, the safety profession has grown significantly and become functionally distinct from related professions such as Human Resources and Project Management. There has even been a drive to make the safety function as independent as possible - see for example the Rogers Commission Report into the Space Shuttle Challenger disaster. The combination of independence and professionalisation of the safety role within organisations leads to the creation of specific safety activities that can be separate from operational work activities.

Because safety practitioners only directly manage and impact the safety-specific activities and objects within their organisation, and because these activities are commonly constructed as 'independent checks' on operational activities, safety practitioners rarely directly affect the design and execution of operational work (Provan et al., 2017). The safety-specific activities are developed, managed and monitored by safety practitioners, and so any reduction in them reduces the authority and importance of those owning, performing and mandating the safety work. Safety activities including safety clutter - are the objects or instruments of authority and relevance for the safety profession.

Safety practitioners are professionally trained in safety management, and they are socialised to think in terms of safety processes, safety systems and safety activities. They are incentivised to add and create new safety programs, and they have the time 
and space within their organisations to think about and create safety work. Safety professionals are also viewed as the professional authority for the appropriateness of this work. Managers and workers rely on the professional authority of safety practitioners as the arbiters of whether a particular activity will improve safety (Almklov, Rosness, \& Størkersen, 2014). Rarely are safety practitioners requested to test and research the efficacy of existing safety activities and almost never are they requested to streamline and remove safety activity as this would could be perceived as line managers either not knowing, or not caring enough about safety.

The combination of training, incentives, and authority leads to a professional identity for safety practitioners which can be described as dogmatic rather than experimentalist (Provan et al., 2018). This dogmatic approach and separation from operational work, can result in the creation and persistence of safety clutter.

The growth and movement of safety professionals between companies and industries creates further opportunities for clutter. The contagion of ideas and activities from organisation to organisation spreads as practitioners are recruited to 'bring across' what they've learned or demonstrated elsewhere. Activities are easier to translate between companies and industries if they are made as generic as possible, leading to safety activities that are insensitive to the specific context of operational work.

\section{Compliance with goal-based regulatory regimes}

As safety regulatory models shift from rule-based to goal-based, organisations have a greater flexibility to set their own rules, processes, and activities for safety (Adams, 2009; Hale, Borys, \& Adams, 2013; Long, Smith, \& Ashhurst, 2016). This flexibility intended to allow organisations to take a context-dependent and efficient approach to safety - instead results in a significant growth of safety activities and safety clutter. 
Under goal-based regulation, managers and safety practitioners need to determine whether they have 'done enough' safety activity to demonstrate the achievement of the performance standard (the 'goal') in the relevant regulation. This test is most often applied retrospectively after the organisation has an accident or incident. The company is then in a position of having a reverse onus of proof, whereby they are guilty until they can prove that they have done everything reasonably practicable. The increase in compliance demands and complexities has coincided with a gradual 'responsibilization' back to organizations themselves (Gray, 2009). Companies are constantly deliberating how much safety activity will be considered enough, on any given day in the eyes of an inspector or a court of law. The cognitive effort expended on such a calculus of accountability has paradoxically been shown to be detrimental to operational goal achievement in other contexts (Lerner \& Tetlock, 1999).

Goal-based legislation and managing safety to a level as low as reasonably practicable creates a standard that is difficult to understand and more difficult to objectively meet. This subjective standard, results in a growth of safety activities and safety clutter, due to the following questions:

- If the safety activity is done elsewhere in the company, why isn't it done everywhere?

- If the activity is done elsewhere in the industry, why isn't it done here?

- If a safety practitioner or safety consultant has recommended it, why aren't you doing it?

- If there is a more expensive but safer way of doing the activity, are you prioritising profit over safety? 
Between 1974 and 2008, Townsend (2013, p. 57) showed a 'mere' doubling of the number of safety statutes, but a hundred-fold increase in regulations interpreting and applying them, with a concomitant proliferation of 'service industries' for safety auditing, researching, pre-qualification, enforcement, publishing, recruitment, training, accreditation and consultancy.

In legal proceedings, it is not enough to do the safety activity, it has to be able to be proven that the activity was done. This creates decision-making and safety activities that are defensible, which is not the same thing as rational. This is clearly demonstrated by the excessive need to document and record safety activities to create a paper-trail of compliance. The focus of these administrative-heavy safety activities is often more about the accurate and timely completion of the paperwork then about the efficacy of the activity for improving the safety of operational work (Johnstone, 2017).

\section{Worrying about safety clutter}

\section{Safety clutter damages employee ownership of safety}

A paradox of modern workplaces is that organisations require their staff to predictably and reliably follow rules, but also to display initiative, flexibility and common-sense (Katz, 1964). The twin objectives can only be achieved at the same time in a world where the rules perfectly match the requirements of every conceivable situation.

Most employees, most of the time, are aware that formal systems of work are not a perfect representation of what is expected from them, and thread a pragmatic line between the undesirable extremes of open disobedience and 'work-to-rule' (or, as the Scandinavians once called it, malicious compliance). Good managers, in turn, might somehow (but seldom explicitly, nor on the record) convey to their employees which rules are important and inflexible, and which rules are context dependent (Amalberti, 
2013). Sociology knows such employees as the 'core set:' the people most closely associated with complex technical systems, who are aware of the ambiguity inherent in their complex environments and unruly technology (Vaughan, 1997, p. 228), who know intimately the 'messy details' of actual practice (Nemeth, Nunnally, O'Connor, Klock, \& Cook, 2005), and who understand 'vernacular safety': the kind of experience, competency and common sense that a formal system based on standardization, centralized control and bureaucratic surveillance is officially blind to (Dekker, 2017; McDonald, Corrigan, \& Ward, 2002).

Safety rules have a peculiar place in this delicate relationship. The very word 'safety' simultaneously signals that a rule is important, and provides a wink and a nod that the rule may be discreetly disobeyed. Safety clutter is in part to blame for this situation. When unproductive work is labelled as 'safety', this reinforces the notion that safety is a constantly spoken value, but not always a practical concern for front-line workers. Under such conditions, workers may pay lip service to safety, but are unlikely to voluntarily engage with safety activities, or to seek out opportunities related to safety - they feel no 'ownership' of the way the organisation has conceptualised their safety.

\section{Safety clutter is bad for adaptability}

Safety management frequently focuses on the need for predictability, seeking to align individual behaviour with organisational safety ideals (Hale \& Borys, 2013). Recently, there has been increased focus on improving spontaneous behaviour. Resilience Engineering (Hollnagel, Woods, \& Leveson, 2006), Safety Differently (Dekker, 2014a), and Safety-II (Hollnagel, 2014) all suggest that local autonomy can be positive for safety. This literature suggests that safety has become overly bureaucratised - that the mechanisms introduced to provide standardisation and control have reduced organisational capacity for safe variation. 
Every piece of safety clutter imposes a time and flexibility constraint on work it reduces the number of different ways that work can be performed without breaking some rule or failing to complete some administrative activity. Much of this reduction in variability is unnecessary - it reduces the number of permissible safe ways to perform the work as much as it reduces the number of unsafe ways. In extreme cases, there may be no way of getting the job done that is both permitted and safe - making it inevitable that either a rule will be broken, or a creatively unsafe situation will be invented. In Study 3, workers lifted gas bottles onto the back of trucks by standing on pallets. The pallets added sufficient extra height to lift correctly following manual handling rules. After an incident where someone rolled their ankle, standing on pallets was banned. There were now two rules, and no possible way to complete the task without breaking one of them.

\section{Safety clutter erodes trust}

Safety clutter usually involves significant amounts of checking. Observations, audits, permits, inspections, and reviews are all activities that are performed primarily to check that other activities have already been performed. Such activities form part of the 'audit explosion' (Power, 2003). Power describes this as 'the rise of 'control of control' in which first-order questions of quality are subordinate to a logic of management system integrity' (2003).

Auditing is necessary for 'reducing asymmetry between agents and principals' (Davies \& Mannion, 1999) - providing governance where less-formal mechanisms are unavailable due to lack of trust or information. Frey (1997), however, suggests that practices that show a lack of trust can encourage the very untrustworthiness they are designed to address. 
Some amount of checking can form an important role in a safety-critical system - it performs a feedback loop to reinforce the likelihood that an important function has been performed. However, what works for mechanical components does not always work for humans. Effective working relationships are built upon a track record of small exchanges of trust (McAllister, 1995). Without the opportunity to be trusted, these exchanges cannot take place.

Safety clutter shifts the focus from 'safety' to 'compliance'. This in turn increases the distance between safety work and operational work (Rae \& Alexander, 2017). Safety work is performed at different times, and by different people, than the operational work it is supposed to help. A key example of this in all of our studies was the amount of time that supervisors spent in offices physically distant from the work they were 'supervising', filling out paperwork that was intended to keep the work safe.

\section{Safety clutter creates an unnecessary trade-off between safety and productivity.}

A site supervisor in Study 3 explained:

“We've lost the plot. It's all about safety by forgetting about productivity and quality. We forget that contractors run a business. People think they are safe because of all the paperwork, where they actually are not. We are here to run a business, not safety."

The more safety activities require time, attention, and expertise, the more they compete with operational work for these resources (Rasmussen, 1997). On Study 4, the cost of personnel time for completing unnecessary safety activities was estimated as three percent of the total project cost (the total cost for completing all safety activities was considerably higher). Additionally, some safety clutter - for example approval processes to use routine tools - posed risks of considerable project schedule delay. 
Safety clutter sets up a situation where the most obvious way to reign in project costs or to recover a slipping schedule is to 'cut corners' on safety. This is not a direct safety problem if the corners cut were unnecessary in the first place - but it is not good for safety in the long term to create a feedback loop whereby surreptitiously avoiding safety activities garners praise and rewards for meeting budgets and deadlines. This is particularly concerning where we don't know, or don't talk about, the difference between necessary and unnecessary safety work.

\section{Dealing with Safety Clutter}

\section{Clutter removal requires an 'evidence-based' approach}

In professions, such as medicine, with a strong evidence-based culture, it is socially acceptable to object to current practices based on evidence that they are ineffective - or even based on a lack of evidence that they are effective (Shojania, Duncan, McDonald, \& Wachter, 2002). Safety is, unfortunately, not such a profession. Despite calls for a more experimental or evidence-based approach to safety practice, most safety practices have never been rigorously evaluated for their effectiveness (Rae, Nicholson, \& Alexander, 2010).

This has a threefold effect on clutter.

Firstly, when someone proposes a new practice, managers and practitioners are not accustomed to demand high quality evidence that the practice is effective.

Secondly, it is difficult to obtain support or funding to test safety practices, and projects to create or introduce practices seldom include substantial evaluation components. This makes it difficult to mount a persuasive case to remove a practice that has already been introduced. 
Thirdly, whilst the standard of evidence required for adding safety activities is very low, a different standard is applied for removing or reducing safety activities. In all of our study organisations, evidence is not demanded for adding activities, but is demanded for removing activities.

In fact, in a climate where asking for evidence is not a routine request, it is difficult to challenge the value of a specific safety activity without appearing to challenge the value of safety itself. Strong safety climates can discourage even wellmotivated skepticism. Managers and safety practitioners are motivated to believe that their current safety practices are effective, or if they are not, the problem is the application of the practice by the workforce that needs correcting, not the practice itself.

Even where there is a widespread belief that a safety practice is ineffective, the practice may be sustained by a small number of influential supporters who sincerely but incorrectly believe that the activity holds value. The lack of systematic evaluation leads to confirmation bias, where a small set of examples of apparent success are used to justify a widespread practice. This is particularly problematic in cases of clutter-bygeneralisation, where there are times and places where the activity is genuinely effective. The lack of systematic evaluation also contributes to attribution bias. If an organisation has a good recent safety record, it is natural to assume that this is because current practices are working. A lack of evidence about what works and what doesn't work in safety, leads to safety clutter being introduced and then not removed.

\section{Specific recommendations}

Safety clutter is not inevitable. For each of the main factors that drive the 'ratchet effect' of clutter, an organisation can take steps to avoid the pressure to add unhelpful safety activity. An organisation can also disengage the ratchet by creating mechanisms for identifying and removing clutter. For an organisation seeking to improve clutter, we 
offer the following suggestions.

\section{Start having conversations about clutter}

The presences of clutter is sustained by social environments in which it is unacceptable to challenge the value of safety, to suggest that some safety activities are a waste of time, or to admit that activities are not carried out in the way that is officially mandated.

The first step in removing clutter is to modify this environment so that specific practices can be challenged without threatening the overall belief in safety. Organisations need to change their language from 'Unless I commit entirely to the company safety philosophy, I don't care enough about safety' to 'I'm challenging the company way of doing this BECAUSE I care about safety'.

The term 'clutter' is, in fact, our own implementation of this first step. We have deliberately introduced this term into our own language, and the language of our industry partners, that gently challenges current practices without attacking the value of safety or the overall approach to safety management.

\section{Find the low hanging fruit}

In our investigations of clutter (for example in Study 4), we use a two-step process for identifying clutter. Firstly, we ask workers directly what they would like to start doing, stop doing, do more of, and do less of, when it comes to safety activity. Secondly, we ask workers to list all of the current safety processes, activities and forms they are involved in, and then ask them what the consequence would be if each activity were stopped. We also encourage managers to ask their workers 'What is the stupidest thing that I am asking you to do to work here every day?' 
Where there is a clear consensus about an item of clutter, there is an opportunity to introduce and test clutter removal without triggering the organisations defense mechanisms that create and preserve clutter.

\section{Conduct a controlled trial for removing a piece of clutter}

Framing clutter removal as a research and evidence problem has several benefits for an organisation.

(1) It requires a discussion about the intended purpose and mechanism of the safety activity. 'We do this to make us safer' is not precise enough for validation; such fuzzy thinking also makes it difficult to have conversations within the organisation generally about the value of safety activities. What is the specific local effect we intend this activity to have? How could this effect be measured? Why do we believe this effect is overall good for safety? Under such questioning the plausibility of many safety activities starts to evaporate, paving the way for clutter removal.

(2) The process of conducting validation fulfils the need individuals feel to be personally responsible for safety, and to be adding value through their actions. Conducting an investigation counts as 'doing something'. It produces a tangible deliverable - knowledge about safety - that is worthy of the time spent.

(3) The existence of evidence answers the 'What if?' and 'documented safety' gap. In response to hindsight challenges about not doing enough for safety, an organisation can produce the evidence for why they were performing some activities and not performing others. 
(4) The successful performance of a small number of controlled trials shifts the burden of proof for safety activities. Once it has been demonstrated that at least some safety activities have zero (or even a negative) impact on safety, it becomes incumbent on anyone proposing a new safety activity to provide evidence that it is not such an activity.

The combined result of these benefits is that testing small amounts of clutter can help an organisation acknowledge to itself that the 'safe decision' is not always to do more safety. We have shown and proposed how adding safety activity can at times cause harm to safety and conversely, removing safety activity can have benefits to safety. Once this threshold concept is realised, there will be a significantly higher burden for adding safety activities, and a significantly lower burden for removing them.

\section{Redefine the role of safety professionals in creating safety}

As the staff in an organisation with most knowledge and influence over the specific structures and processes for administering safety systems, safety professionals shoulder most responsibility for removing clutter. There are three specific areas in which safety professionals can adjust their own practice in response to clutter.

Firstly, external stakeholders make demands that may directly result in clutter. They may:

- insist on rapid action in response to an incident or safety concern;

- ask for 'demonstration' of safety, through the documentation of decision making;

- require over-specification and over-generalisation of safety-related processes; and

- overlap with other stakeholders' requests that results in duplication. 
Safety professionals cannot control the behaviour of external stakeholders. Safety professional actions can, however, reduce the impact of these demands on the organisation through actively managing these stakeholders, or multiply the impact of the demands by passively creating internal compliance mechanisms that match or exaggerate the stakeholder compliance mechanisms. Whilst companies complain about external rules, most compliance activity is self-imposed.

Secondly, senior managers look to their safety function for advice on how to demonstrate safety leadership. Safety professionals have an opportunity to promote leadership styles that focus on asking open questions, collecting evidence, and showing interest in operational work, rather than attempting to 'add value' by taking direct part in low-level safety activities.

There is a direct trade-off for a manager between taking personal responsibility for safety by specifying safety activities, and empowering others to take responsibility for safety. Both are legitimate safety values, but organisations should make the choice in full knowledge that focussing on management responsibility for safety reduces worker ownership of safety.

Thirdly, safety professionals can take a reflective, evidence-based approach to their own practice. A key part of this is recognising that something going wrong is not evidence that something needs to be done - and is certainly not evidence for any specific action.

There will often be occasions with social and psychological pressure to take rapid action to improve safety. Actions taken at such times, unless backed up by evidence, are very likely to create safety clutter. 
By taking this approach, safety professionals will model a mature safety attitude to the rest of their organisation. It does not reflect poor safety culture, or poor individual attitude, to challenge the value of a specific safety activity. Concern about the efficacy of, and evidence for, specific practices is a good thing for safety.

\section{Conclusion and Further Work}

In this paper we presented a qualitative description of safety clutter - the accumulation of safety procedures, documents, roles and activities that are performed in the name of safety, but do not contribute to the safety of operations. In addition to the opportunity cost, safety clutter is harmful because it damages ownership and trust, it is bad for adaptability, and it unnecessarily harms productivity.

We described three main mechanisms by which clutter is generated:

- Duplication - multiple activities fulfilling the same safety function

- Generalisation - applying safety rules beyond the context in which they are useful

- Over-specification - unnecessarily proceduralisation of work in the name of safety

We also suggested that these mechanisms arise from a ratchet effect, coming from two asymmetries:

(1) It is easier to add or expand safety work, than to remove or reduce safety work.

(2) There are many regular or ad-hoc events that trigger the addition or expansion of safety work, but relatively few opportunities to remove or reduce safety work.

Up to this point, our findings are strongly grounded in theory and data collected through 4 separate research studies. More tentatively, we suggested several underlying 
causes of the asymmetries that drive safety clutter. These causes are also forces that drive safety improvement:

(1) Responses to accidents and incidents that trigger additional safety work

(2) A need to demonstrate to others that we are managing safety

(3) The separation and professionalisation of the safety role

(4) Compliance with goal-based regulatory regimes

Based on our discussion in this paper, there are two open questions deserving more rigorous empirical investigation.

Firstly, what is the relationship between safety practitioners and safety clutter? Does adding safety staff to an organisation increase the amount of clutter? If so, is there an optimal staffing level beyond which further safety staff cause more harm than benefit?

Secondly, what is the relationship between senior leadership commitment to safety and clutter? In particular, does strong leadership commitment (for example, a Zero Accident Vision) result in minor safety issues being managed at higher levels within an organisation, leading to increased clutter?

More generally, and less amenable to direct empirical investigation, is reduction of clutter a desirable goal? Whilst the negative consequences of clutter are well evidenced, this does not necessarily mean that explicit attempts to reduce clutter will yield corresponding benefits. In a complex system there is no such thing as a 'simple' intervention with predictable consequences.

\section{Bibliography}


Almklov, P. G., Rosness, R., \& Størkersen, K. (2014). When safety science meets the practitioners: Does safety science contribute to marginalization of practical knowledge? Safety Science, 67(Supplement C), 25-36. https://doi.org/10.1016/j.ssci.2013.08.025

Amalberti, R. (2013). Navigating Safety: Necessary Compromises and Trade-Offs Theory and Practice (2013 edition). New York: Springer.

Bourrier, M., \& Bieder, C. (2013). Trapping Safety into rules: an introduction. Trapping Safety into Rules. How Desirable or Avoidable Is Proceduralization. Ashgate, Farnham, 1-9.

Davies, H. T. O., \& Mannion, R. (1999). Striking a Balance Between Checking and Trusting (The York Series on the NHS White Paper No. Discussion Paper 165). York: Centre for Health Economics.

Dekker. (2017). The Safety Anarchist: Relying on human expertise and innovation, reducing bureaucracy and compliance. New York: Routledge.

Dekker, S. (2014a). Safety Differently: Human Factors for a New Era, Second Edition (2 edition). Boca Raton: CRC Press.

Dekker, S. (2014b). The bureaucratization of safety. Safety Science, 70, 348-357.

Frey, B. S. (1997). Not just for the money: an economic theory of personal motivation. Cheltenham: Edward Elgar Publishing.

Hale, A., \& Borys, D. (2013). Working to rule, or working safely? Part 1: A state of the art review. Safety Science, 55, 207-221.

Hollnagel, E. (2014). Safety-I and Safety-II (New edition edition). Farnham, Surrey, UK England; Burlington, VT, USA: Ashgate.

Hollnagel, E., Woods, D. D., \& Leveson, N. (Eds.). (2006). Resilience Engineering: Concepts and Precepts (New edition). Ashgate Publishing Limited. 
Howard-Payne, L. (2015). Glaser or Strauss? Considerations for selecting a grounded theory study: South African Journal of Psychology. https://doi.org/10.1177/0081246315593071

Johnstone, R. E. (2017). Glut of Anesthesia Guidelines a Disservice, Except for Lawyers. Anesthesiology News, 42(3), 1-6.

Leape, L. L. (2014). The Checklist Conundrum. New England Journal of Medicine, 370(11), 1063-1064. https://doi.org/10.1056/NEJMe1315851

Manuele, F. A. (2009). Leading \& Lagging Indicators. Professional Safety, 54(12), 28 33.

McAllister, D. (1995). Affect- and Cognition-Based Trust as Foundations for Interpersonal Cooperation in Organizations. The Academy of Management Journal, 38(1), 24--59.

McDonald, N., Corrigan, S., \& Ward, M. (2002). Well-intentioned people in dysfunctional systems. In Keynote Presented at Fifth Workshop on Human Error, Safety and Systems Development, Newcastle, Australia.

McGhee, G., Marland, G. R., \& Atkinson, J. (2007). Grounded theory research: literature reviewing and reflexivity. Journal of Advanced Nursing, 60(3), 334342. https://doi.org/10.1111/j.1365-2648.2007.04436.x

Nemeth, C., Nunnally, M., O’Connor, M., Klock, P. A., \& Cook, R. (2005). Getting to the point: developing IT for the sharp end of healthcare. Journal of Biomedical Informatics, 38(1), 18-25. https://doi.org/10.1016/j.jbi.2004.11.002

Pink, D. (2011). Drive: The Surprising Truth About What Motivates Us. New York: Riverhead Books.

Power, M. (2003). Evaluating the Audit Explosion. Law \& Policy, 25(3), 185-202. https://doi.org/10.1111/j.1467-9930.2003.00147.x 
Provan, D. J., Dekker, S. W. A., \& Rae, A. J. (2017). Bureaucracy, influence and beliefs: A literature review of the factors shaping the role of a safety professional. Safety Science, 98(Supplement C), 98-112. https://doi.org/10.1016/j.ssci.2017.06.006

Provan, D. J., Dekker, S. W. A., \& Rae, A. J. (2018). Benefactor or burden: Exploring the professional identity of safety professionals. Journal of Safety Research. https://doi.org/10.1016/j.jsr.2018.05.005

Rae, A. J., \& Alexander, R. D. (2017). Probative blindness and false assurance about safety. Safety Science, 92, 190-204. https://doi.org/10.1016/j.ssci.2016.10.005

Rae, A. J., Nicholson, M., \& Alexander, R. D. (2010). The State of Practice in System Safety Research Evaluation. In IET System Safety Conference. Manchester.

Shojania, K. G., Duncan, B. W., McDonald, K. M., \& Wachter, R. M. (2002). Safe but Sound: Patient Safety Meets Evidence-Based Medicine. JAMA, 288(4), 508513.

Stock, C. T., \& Sundt, T. (2015). Timeout for Checklists? Annals of Surgery, 261(5), $841-842$.

Townsend, A. S. (2013). [(Safety Can't be Measured: An Evidence-Based Approach to Improving Risk Reduction)]. Gower Publishing Ltd.

Vaughan, D. (1997). The Challenger Launch Decision: Risky Technology, Culture, and Deviance at NASA (1 edition). Chicago: University Of Chicago Press.

Wears, R. L., \& Hunte, G. S. (2014). Seeing patient safety “like a state.” Safety Science, $67,50-57$.

Weber, D., Macgregor, S., Provan, D. J., \& Rae, A. J. (2018). "We can stop work, but then nothing gets done." Factors that support and hinder a workforce to discontinue work for safety. Safety Science, 108C, 149-160. 
